\title{
Photospheric physical conditions before a two-ribbon solar flare
}

\author{
S. N. Chornogor $\dagger$ and N. N. Kondrashova \\ Main Astronomical Observatory, National Academy of Sciences of Ukraine, \\ 27 Akademika Zabolotnogo St., 03680 Kyiv, Ukraine \\ email: chornog@mao.kiev.ua
}

\begin{abstract}
We present preliminary results of the study of the photospheric physical conditions about 30 minutes before the $2 \mathrm{~N} / \mathrm{M} 2$ two-ribbon solar flare. The soft X-ray enhancement, $H_{\alpha}$ brightening and microwave bursts occured prior to the flare. We demonstrate the semiempirical models of the preflaring photosphere fot two kernels of the future flare. These models derived from the inversion reproduce the spectral observations in seven Fraunhofer lines. The models consist of two atmospheres: the magnetic flux tube and its nonmagnetic surroundings. The inferred models show strong changes of the photospheric physical conditions in the preflare phase. The preheating was found in the flux tube. Upward motions of the surrounding photospheric material were revealed. Significant discrepancies of the flux-tube temperature and density are found for two sites of the future flare. The inner kernel located near the filament was cooler in the photosphere than the outer kernel.
\end{abstract}

Keywords. Sun, active region, flares, photosphere, models

One of the most complicated problems in solar flare research is understanding a flare build-up. It is now widely accepted that the flare energy is stored in the current sheets, and the fast reconnection leads to its eruptive release. The reconnection of the largescale magnetic fields may be triggered by the preflare reconnection of the magnetic field occurred near the magnetic neutral line at the lower atmosphere (see for example Zhang et al. 2000; Moon et al. 2004) Alikaeva et al. (1993) revealed the difference between physical conditions in the photosphere in active regions prior to and in the absence of flares

We have studied the preflare physical state of the photosphere in two sites about 30 minutes before the 2N/M2 solar two-ribbon flare that occurred on June 3, 1979 in McMath 16051. The active region showed a complex multipolar magnetic congiguration and had high flare activity. The new magnetic fluxes appeared along the polarity inversion line (Golovko \& Kotrc (1992)). The flare occurred at 08:42 UT and lasted for 2.5 hours. The preflare activity was characterized by soft X-ray enhancement, $H_{\alpha}$ brightening and radio bursts.

We uzed the spectrum of the active region obtained at 08:09 UT with the solar horizontal telescope of the Main Astronomical Observatory (Kyiv, Ukraine). The spectrograph slit intersected one of the ribbons of the future flare. The brightening in the $H_{\alpha}$ line was observed in two kernels A and B. The kernel B was located near the filament lying between the ribbons. The soft X-ray burst was assotiated with the $H_{\alpha}$ brightening. The moment of the spectral observations approximately coincides with its maximum. Preflare soft X-ray enhancement, $H_{\alpha}$ brightening and radio bursts probably were the flare precursors.

$\dagger$ Present address: Main Astron. Observ., 27 Akademika Zabolotnoho St., 03680 Kyiv, Ukraine. 


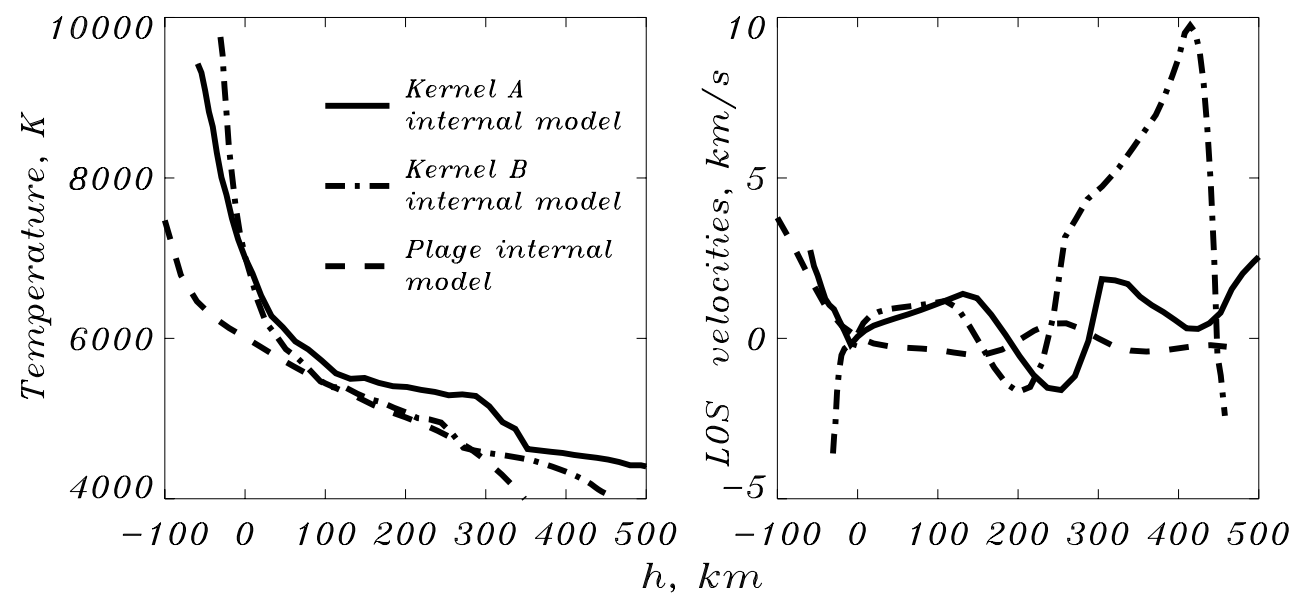

Figure 1. The temperature and LOS velocity runs of the internal models for kernel A (solid line) and kernel B (dash-dotted line). For comparison, the temperature and LOS velocities of the plage internal model of Bellot Rubio et al. are depicted as well (dashed line). Positive values indicate downflows.

Our modelling is based on seven Fraunhofer lines. They are formed in a wide height interval. All the lines were changed before the flare. They were weakened and blueshifted relative to the undisturbed photosphere lines. The line-of-sight velocities of the upflows were equal to $0.5-2 \mathrm{~km} / \mathrm{s}$. The SIR (Stokes Inversion) code used for the modelling has been described in Ruiz Cobo \& Toro Iniesta (1992). Our models include two components: a thin magnetic flux tube and nonmagnetic surroundings. The plage internal model of Bellot Rubio et al. (2000) was employed as an initial flux-tube model component. The Harvard Smithsonian Reference Atmosphere (Gingerich et al. 1971) has been adopted for the ambient medium as an initial model. Figure 1 shows the temperature and LOS velocity runs of the inferred internal models for kernels A and B.

The main results are the following.

(1) The inferred models show the difference of the preflaring physical state from that of undisturbed plage atmosphere. The preheating and gas pressure excess are found in the flux tubes.

(2) The external medium is characterized by upflows. The internal models reveal the discrepancies between the line-of-sight velocities in the kernels A and B. Strong downflows in the kernel $\mathrm{A}$ and upflows in the inner kernel $\mathrm{B}$ are found below $\mathrm{h}=0 \mathrm{~km}$.

(3) The macroturbulent motions in the flux tubes of both kernels are suppressed before the flare.

\section{References}

Alikaeva, K.V., Kondrashova, N.N., Redyuk, T.I. \& Rudnikova, E.G. 1993, KFNT 9, 57.

Bellot Rubio, L.R., Ruiz Cobo, B. \& Collados, M. 2000, ApJ 535, 475.

Gingerich, O., Noyes, R.W., Kalkofen, W. \& Cuny, Y. 1971, Sol.Phys. 18, 347.

Golovko, A.A. \& Kotrc, P. 1992, Sol.Phys. 142, 67.

Moon, Y.-J., Chae, J., Choe, G.S., et al. 2004, JKAS 37, 41.

Ruiz Cobo, B. \& Toro Iniesta, J.C. 1992, ApJ 398, 375.

Zhang, H.Q., Sakurai, T., Shibata, K. et al. 2000, A\&A 357, 725. 